\title{
Dual-fluorescence pH probe for bio-labelling $\dagger$
}

Cite this: Phys. Chem. Chem. Phys. 2015, 17, 30590

Received 12th September 2015, Accepted 16th October 2015

DOI: $10.1039 / \mathrm{c} 5 \mathrm{cp} 05454 \mathrm{k}$

www.rsc.org/pccp

\author{
C. Richter, ${ }^{a}$ C. Schneider, ${ }^{\text {b }}$ M. T. Quick, ${ }^{a}$ P. Volz, ${ }^{\text {b }}$ R. Mahrwald, ${ }^{a}$ J. Hughes, ${ }^{c}$ \\ B. Dick, ${ }^{d}$ U. Alexiev ${ }^{\star b}$ and N. P. Ernsting ${ }^{* a}$
}

\begin{abstract}
Although seminaphtorhodafluor (SNARF) dyes are already widely used to measure $\mathrm{pH}$ in cells and at biofilms, their synthesis has low yield and results in an unspecific position of a carboxy-group. The separation of $5^{\prime}$ - and $6^{\prime}$-carboxy-SNARF reveals a $\mathrm{p} K_{\mathrm{a}}$ difference of 0.15 , calling into question $\mathrm{pH}$ measurements with the (commercially available) mixture. Here we replace the bulky external dicarboxyphenyl ring with a propionate group and evaluate the spectral properties of the new derivative. Proceeding to the ethyl-iodoacetamide, covalent linkage to cysteine protein sites is achieved efficiently as shown with a cyanobacterial phytochrome, extending the scarce application of SNARF in bio-labelling in the current literature. Application in fluorescence lifetime imaging is demonstrated both with the lifetime-based and ratiometric-yield method.
\end{abstract}

\section{Introduction}

Xanthene-based ratiometric pH-probes have been used extensively in the investigation of $\mathrm{pH}$ in cells and at biofilms. ${ }^{1}$ Fluorescein homologues are most commonly used, for example BCECF (Scheme 1) which was optimized for solubility. ${ }^{2-4}$ However, only their emission intensity and not the color is affected by protonation,
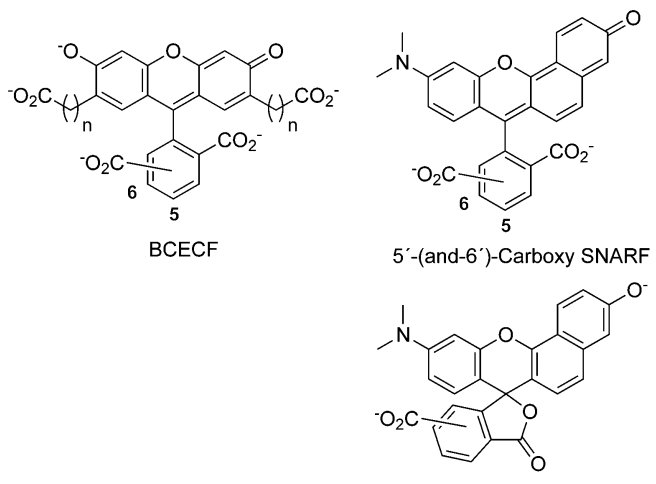

Lacto form

Scheme 1 Chemical structures of xanthene-based $\mathrm{pH}$-probes.

\footnotetext{
${ }^{a}$ Department of Chemistry, Humboldt-Universität zu Berlin, 12489 Berlin, Germany. E-mail: nernst@chemie.hu-berlin.de

${ }^{b}$ Department of Physics, Freie Universität Berlin, 14195 Berlin, Germany. E-mail: ulrike.alexiev@fu-berlin.de

${ }^{c}$ Institute for Plantphysiology, Justus-Liebig Universität, 35390 Giessen, Germany

${ }^{d}$ Institute for Physical and Theoretical Chemistry, Universität Regensburg, 93053 Regensburg, Germany

$\dagger$ Electronic supplementary information (ESI) available: Synthesis and characterization of all compounds can be found. See DOI: $10.1039 / \mathrm{c} 5 \mathrm{cp} 05454 \mathrm{k}$
}

so that ps-time-resolved measurements of the fluorescence decay are often needed in quantitative $\mathrm{pH}$ experiments. For imaging one would prefer a dual emission band which reflects the protonated and deprotonated populations more directly. This distinction can be achieved with "seminaphtorhodafluor" homologues like carboxy-SNARF. ${ }^{5-14}$ Advantages of these dyes include a wide excitation range, fluorescence from both protonated and deprotonated forms, and a $\mathrm{p} K_{\mathrm{a}}$ between them which is in the physiological range.

Several drawbacks currently prevent more widespread use of the SNARF probes mentioned above, however. (i) Their synthesis has low yield and results in an unspecific position $\left(5^{\prime}\right.$ or $\left.6^{\prime}\right)$ of a second carboxy-group..$^{5-7}$ Note that the isomeric mixture is commonly used in the literature. ${ }^{13-15}$ (ii) Available spectral and photophysical data are less extensive than for fluorescein. For example, in the equivalent case of $5^{\prime}$ - and $6^{\prime}$-carboxyfluorescein the differences have been described; ${ }^{16,17}$ such a distinction is still missing for rhodafluors. (iii) At higher $\mathrm{pH}$ the dicarboxyphenyl ring, being external to the electronic chromophore, causes a lactonic form and therefore renders a fraction of the dissolved dye useless. ${ }^{18-20}$

Here we report the new derivatives PAc- and EA-SNARF (Scheme 2) where the problematic dicarboxyphenyl ring has been replaced by a propionate or ethylamino group, respectively. For investigations of protonation dynamics near the entrance or exit site of proton channels in proteins, for example, covalent linkage of the dye to a target site is needed. A widely-used linker for cysteines is maleimide. The connection of maleimide to the SNARF-system was already described by I. Himachi and coworkers, ${ }^{7}$ but their chain is quite long and, correspondingly, the footprint of the tethered dye is too large for spatial resolution of the proton wire topology at the protein surface. To overcome 

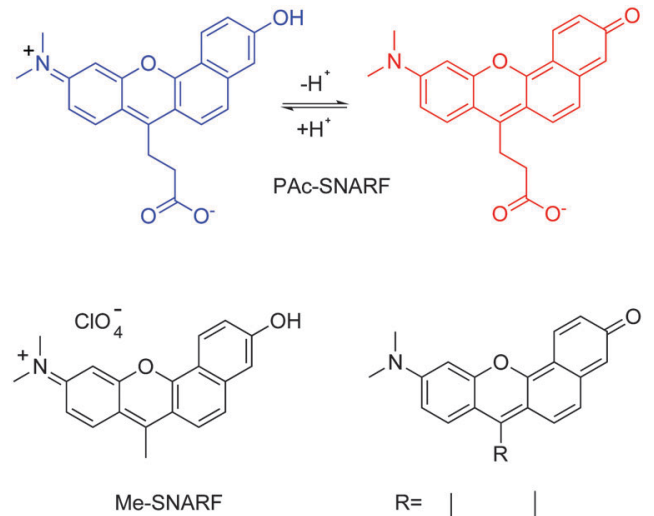$$
\text { Me-SNARF }
$$

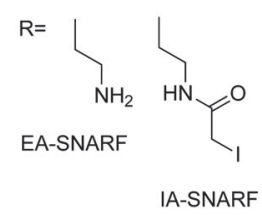

Scheme 2 New derivatives with propionate (PAC), ethyl-amino (EA) and iodoacet-amide (IA) functionality. Colors highlight the spectral difference.

this limitation we developed IA-SNARF (see also Scheme 2), described here, where the fluorescent core bears an ethyl chain with an iodacetamido group. This allows us to label a cyanobacterial phytochrome covalently at a free cysteine. Spectral and radiative properties of the label are described. This novel SNARF with dual pH-dependent fluorescence bands and wellseparated fluorescence lifetimes is better suited for imaging than fluorescein derivatives. ${ }^{7,21-23}$

\section{Experimental section}

Syntheses are described in the ESI. $\dagger$

Solutions. Measurements were carried out at $\mathrm{pH}$ 5-9 as measured with a glass electrode. Generally dimethyl sulfoxide (dmso, spectrograde from Merck) was used as cosolvent; the concentration was water:dmso 20:1 (vol:vol before mixing) unless stated otherwise. For stationary measurements, standard buffer solutions from Merck were diluted with water $1: 1$. Note that the dmso cosolvent shifts the $\mathrm{pH}$ up by 0.1 on average. Buffers consisted of a disodium-hydrogenphosphate/potassiumdihydrogenphosphate mixture or of a citric acid/sodium hydro$\mathrm{xyl} /$ hydrochloric acid mixture (traceable to SRM from NIST and PTB.) The sodium salt of the carboxy-derivatives PAc- and $5^{\prime}$ (or 6') C-SNARF dissolves excellently in water, but after acidification the absorption spectra at $\mathrm{pH} \sim 5$ showed amplitude variations if the peak absorbance of the protonated form exceeded 0.2 over $1 \mathrm{~cm}$. The variations are possibly caused by very slow aggregation or other adsorption processes which we cannot account for. Thus it is challenging to obtain optical spectra with a well-defined isosbestic point. For time-resolved fluorescence measurements, the sodium salt of PAc-SNARF was first dissolved in Tris-citrate buffer (Sigma) at $\mathrm{pH}$ 10; subsequent titration down to $\mathrm{pH} 5$ resulted in absorbance spectra with clear isosbestic points.

Quantum-chemical calculations were performed with timedependent density functional theory (TD-DFT), and ground state geometries were optimized with density functional theory (DFT). ${ }^{24}$ The B3LYP functional and the cc-pVDZ atomic basis set were used throughout. Solvents effects were taken into account with the polarized continuum (PCM) model.

Optical spectra. Absorption spectra were measured with a Cary 300 Bio and a Shimadzu UV2450 UV-visible spectrophotometer. Fluorescence spectra were recorded with a Spex Fluorolog 212 and a Spex Fluoromax. Excitation wavelengths were 488, 526 and $545 \mathrm{~nm}$; the latter two are at or close to the isosbestic point of absorption by PAc-SNARF. Raw fluorescence spectra were multiplied with a photometric correction function; in this way the relative number of photons/(s nm), or quantum distribution $F(\lambda)=\partial \Phi / \partial \lambda$, is obtained for each emission wavelength $\lambda$. The bandwidth was generally $2 \mathrm{~nm}$, and wavelengths were checked with $\mathrm{Hg}$ lines.

The quantum yield of fluorescence from PAc-SNARF was determined at $\mathrm{pH}$ 9, i.e. for the deprotonated form. For this purpose the dmso concentration was adjusted so that the peak absorbance wavelength and value of the solution coincide with that of a solution of rhodamine 101 in methanol. Following excitation at the peak ( $1 \mathrm{~nm}$ bandwidth), the fluorescence bands were recorded and photometrically corrected as before. The band integrals are related like the quantum yields, which for rhodamine 101 is $1.0 .^{25}$ The entire experiment, starting with the preparation of both solutions, was repeated three times.

Fluorescence decay curves were measured with a tunable picosecond laser in a TCSPC setup which is based on a microchannel plate. ${ }^{26,27}$ Sample fluorescence was excited by a whitelight laser together with an acusto-optical tunable filter (SuperK Extreme EUV3 and SELECT UV-VIS, NKT). The output power was $140 \mu \mathrm{W}(488 \mathrm{~nm})$ and $220 \mu \mathrm{W}(526 \mathrm{~nm})$ at $19.5 \mathrm{MHz}$ repetition rate. Emission was spectrally selected either with a $580 \mathrm{~nm}$ or $630 \mathrm{~nm}$ interference filter (Edmund 47139 THGC and EO65706 $580 \mathrm{~nm}$ 1-9-13), or else with a long-pass filter (LP) $\geq 545 \mathrm{~nm}$ (HQ545, Chroma) or LP 715 nm (RG715, Schott). Fluorescence decay traces were collected in 1024 time channels with a channel width of $19.5 \mathrm{ps}$. The instrumental response function (IRF) was determined at the corresponding wavelengths with a colloidal silica solution as scattering material (LUDOX, Grace). The IRF of the system had 60-70 ps full width at half maximum. After deconvolution with the IRF the traces were fitted by a sum of exponentials. $^{26,28}$

Fluorescence lifetime imaging microscopy (FLIM) ${ }^{29}$ was performed with the same laser system. The setup consists of an inverted microscope (Olympus IX71), equipped with a $4 \times$ or $40 \times$ objective, and a confocal scanning unit (DCS-120, Becker \& Hickl). Time-correlated single photon counting was performed with a corresponding module (SPC-160, Becker \& Hickl) and a hybrid PMT (HPM-100-40, Becker \& Hickl). The time range was set to 19.97 ns for 1024 channels. For ratiometric-yield experiments the excitation was at $526 \mathrm{~nm}$ and emission was spectrally selected by a dichroic filter 560 dclp for the dual detection mode. In the "green" and "red" emission channel an additional HQ545LP, respectively OG590 longpass filter, was used. For lifetime-based analyses the same settings as in the spectroscopic experiments were used. Additionally, excitation and filter settings are given in the text. 
SNARF labelling of cyanobacterial phytochrome. The Cph1 $\Delta 2$ sensory module ${ }^{30}$ was labelled with a 5 -fold excess of IA-SNARF in $2.5 \mathrm{mM}$ Tris $\mathrm{pH} 7.8,15 \mathrm{mM} \mathrm{NaCl}$ for 1 hour at room temperature. Unbound dye was removed by gel filtration (Sephadex G-25 fine, GE Healthcare). The labelling stoichiometry was calculated according to

$$
\frac{c_{\text {Label }}}{c_{\text {Protein }}}=\left(\frac{\Delta A_{\mathrm{L}}}{\varepsilon_{\mathrm{L}}}\right)\left(\frac{\varepsilon_{\text {Protein }}}{A_{\text {Protein }}}\right) \text {. }
$$

Here $A_{\text {Protein }}$ is the absorbance at $658 \mathrm{~nm}\left(\lambda_{\max }\right)$ of Cph1 $\Delta 2$ in the Pr state at $\mathrm{pH} 8.8,15 \mathrm{mM} \mathrm{NaCl}$. We determined the corresponding extinction coefficient, $\varepsilon_{\text {Protein }}$, to be $\sim 500001 \mathrm{~mol}^{-1} \mathrm{~cm}^{-1}$ under our conditions, significantly lower than that reported by van Thor et al. ${ }^{32} \Delta A_{\mathrm{L}}$ is the absorbance of the label, measured as the absorbance difference between the labelled and unlabelled phytochrome at $565 \mathrm{~nm}$. The extinction coefficient of SNARF at $565 \mathrm{~nm}$ (deprotonated form) is $\varepsilon_{\mathrm{L}}=47100 \mathrm{l} \mathrm{mol}^{-1} \mathrm{~cm}^{-1}$, as described in the results section. Covalent binding of the IA-SNARF label to Cph1 $\Delta 2$ and the removal of excess label was verified by the fluorescence of the Cph1 $\Delta 2$ band following SDSPAGE. The ability of the SNARF-labelled phytochrome to shift between the far red (Pfr) and red (Pr) form was tested by illumination at $\lambda=735 \mathrm{~nm}$. The phytochrome samples remained fully photochromic after derivatisation, indicating that the procedure did not affect molecular function.

Fluorescence imaging of HeLa cells was performed in phosphate-buffered saline (PBS) at $\mathrm{pH}$ 7.3. Cells were incubated with PAc-SNARF and IA-SNARF, respectively, for 30 minutes and subsequently washed with PBS. Incubation and imaging were carried out at $37^{\circ} \mathrm{C}$.

\section{Results and discussion}

\subsection{Properties of PAc- and other SNARF derivatives}

Stationary absorption and emission spectra are shown in Fig. 1 as function of pH. Spectra have been scaled to the same sample concentration. In absorption an isosbestic point is observed at $526 \mathrm{~nm}$. At shorter wavelengths the extinction coefficient of the protonated form exceeds that of the deprotonated form. In stationary measurements of fluorescence, in order to enhance the relatively weak "blue" emission from the protonated species, we excite at $\lambda_{\text {exc }}=488 \mathrm{~nm}$. The appearance of the spectra is very similar to that of the known $5^{\prime}, 6^{\prime} \mathrm{C}$-SNARF mixture.

For analysis we may assume that the protonated and deprotonated forms are in equilibrium in the electronic ground state. The absorption spectra in Fig. 1a can therefore be decomposed into contributions from the two ground-state forms to yield the species-associated spectra (SAS). These are shown as red and blue lines, respectively, in panel (a). Their relative weight, corresponding to the mole fractions $\mathrm{x}$ of the two forms, is described by

$$
x_{\text {red }}=10^{-\mathrm{p} K_{\mathrm{a}}} /\left(10^{-\mathrm{p} K_{\mathrm{a}}}+10^{-\mathrm{pH}}\right) \text { and } x_{\text {blue }}=1-x_{\text {red }} .
$$

A fit to the data gives $\mathrm{p} K_{\mathrm{a}}=7.55( \pm 0.12$ standard error $)$ for the protonation equilibrium, as measured by absorption.
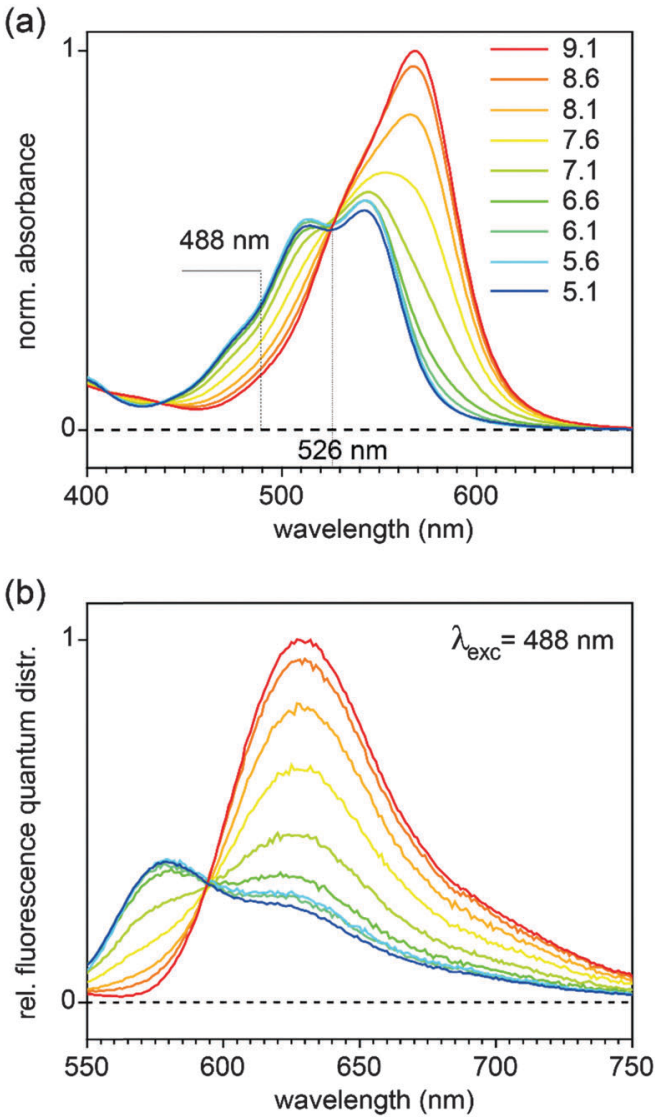

Fig. 1 Optical absorption (a) and fluorescence (b) spectra of PAc-SNARF in water/dmso ( $20: 1$ by volume, for buffer see text) as function of $\mathrm{pH}$ at $22{ }^{\circ} \mathrm{C}$. Red and blue curves represent the species-associated spectra (SAS) of the pure deprotonated and protonated species, respectively, from global analysis. In stationary measurements, excitation wavelength $\lambda_{\mathrm{exc}}=$ $488 \mathrm{~nm}$ was chosen in order to enhance the fluorescence contribution by the protonated species.

The fluorescence spectra in Fig. 1b, when treated in the same manner, give a similar $\mathrm{p} K_{\mathrm{a}}=7.50( \pm 0.20)$. The resulting SAS are shown as a red and blue lines, respectively, in panel (b).

The maximum extinction coefficient of the deprotonated form $\varepsilon_{\text {red,max }}=47100 \pm 500 \mathrm{l} \mathrm{mol}^{-1} \mathrm{~cm}^{-1}$ was determined at $\mathrm{pH} 9 .^{31}$ From Fig. 1a we find that upon protonation the maximum extinction coefficient drops to $58 \%$, hence $\varepsilon_{\text {blue,max }}=27320 \mathrm{l} \mathrm{mol}^{-1} \mathrm{~cm}^{-1}$.

Species-associated spectra were fitted by one or several functions

$$
\log \operatorname{norm}(\tilde{\nu})=h \exp \left\{-\ln 2\left(\frac{\ln \left[1+2\left(\tilde{\gamma}-\tilde{\gamma_{\mathrm{p}}}\right) / \Delta\right]}{\gamma}\right)^{2}\right\}
$$

With parameters $\widetilde{\nu_{\mathrm{p}}}, \Delta, \gamma, h$ for peak wavenumber $\left[\mathrm{cm}^{-1}\right]$, width $\left[\mathrm{cm}^{-1}\right]$, asymmetry [-], and amplitude [-]. Note that in case of absorption, $h$ refers to extinction coefficients (in $1 \mathrm{~mol}^{-1} \mathrm{~cm}^{-1}$ ). In case of fluorescence, quantum distributions over wavelengths were converted into distributions over wavenumbers, $F(\tilde{\nu})=(\partial \Phi /(\Phi \tilde{\nu})) \propto(\partial \Phi / \partial \lambda) \lambda^{2}$ and then set to 1 at the peak of the spectrum of the deprotonated form $(\Phi$ is the total number of 
Table 1 Lognormal description of $\mathrm{S}_{1}-\mathrm{S}_{0}$ bands of PAc-SNARF in water/ dmso (20:1 vol: vol)

\begin{tabular}{|c|c|c|c|c|}
\hline & $\widetilde{\nu_{\mathrm{p}}} / \mathrm{cm}^{-1}$ & $\Delta / \mathrm{cm}^{-1}$ & $\gamma$ & $h$ \\
\hline \multicolumn{5}{|c|}{ Absorption (Fig. 1a) } \\
\hline \multirow[t]{3}{*}{ Deprotonated $^{a}$} & 16309 & 971 & -0.42 & 3425 \\
\hline & 17602 & 1576 & 0.19 & 47100 \\
\hline & 19033 & 1675 & 0.49 & 15926 \\
\hline \multirow[t]{4}{*}{ Protonated $^{b}$} & 16934 & 1450 & -0.58 & 563 \\
\hline & 18206 & 974 & -0.07 & 13403 \\
\hline & 19130 & 1696 & -0.25 & 23321 \\
\hline & 20385 & 2012 & 0.53 & 13808 \\
\hline \multicolumn{5}{|c|}{ Emission $^{c}$ from $488 \mathrm{~nm}$ excitation (Fig. 1b) } \\
\hline \multirow[t]{2}{*}{ Deprotonated } & 15844 & 1605 & -0.12 & 1.000 \\
\hline & 14174 & 1434 & -0.25 & 0.193 \\
\hline \multirow[t]{3}{*}{ Protonated } & 17283 & 1219 & 0.03 & 0.316 \\
\hline & 15953 & 1604 & -0.26 & 0.247 \\
\hline & 14242 & 1562 & -0.42 & 0.044 \\
\hline
\end{tabular}

photons counted). Log norm parameters are collected in Table 1 and Tables S1-S5 (ESI $\dagger$ ).

Fluorescence lifetime curves are shown and analyzed in Fig. 2. Fluorescence time traces were recorded at different $\mathrm{pH}$ values in Tris-citrate buffer (panel a). In these time-resolved fluorescence measurements the sample was excited at the isosbestic point (526 nm), ensuring the same excitation probability for the deprotonated and protonated form, and emission was detected at $\lambda>545 \mathrm{~nm}$. In line with the considerations above we assume that each species possesses an individual fluorescence lifetime that is constant over the $\mathrm{pH}$ range. The relative amplitudes of the two global lifetime components $\left(\tau_{\text {red }}=2.21 \mathrm{~ns}\right.$ for the deprotonated "red" form and $\tau_{\text {blue }}=0.49 \mathrm{~ns}$ for the protonated "blue" form) are shown as data points in Fig. 2b. A fit gives $\mathrm{p} K_{\mathrm{a}}=7.55( \pm 0.02)$. Similarly, the amplitude-weighted lifetime displays the expected behavior with $\mathrm{pH}$ (panel c) and we find $\mathrm{p} K_{\mathrm{a}}=7.54( \pm 0.03)$. Note that these lifetime-based analyses are independent of the dye concentration.

The dependence of fluorescence lifetimes on excitation and emission wavelengths is shown in Fig. 3. At pH 10 the deprotonated form is detected easily as a pure species with $2.21 \mathrm{~ns}$ lifetime ( $\lambda_{\mathrm{ex}}=526 \mathrm{~nm}, \lambda_{\mathrm{em}}=545 \mathrm{~nm}$ long pass). The same observation is made even upon excitation at the "blue" side of the absorption spectrum $\left(\lambda_{\mathrm{ex}}=488 \mathrm{~nm}\right)$, by choosing an appropriate emission filter (e.g. longpass $715 \mathrm{~nm}$ or bandpass $630 \mathrm{~nm}$ ). At $\mathrm{pH} 5$ the protonated form, when excited at the isosbestic point or at $488 \mathrm{~nm}$, gives fluorescence decay traces with a main decay component of $0.49 \mathrm{~ns}$ (96-97\%). The remaining percentages can be attributed to the residual amount of the deprotonated form, in particular when detecting in the "red" emission band $\left(\lambda_{\text {ex }}=488 \mathrm{~nm}, \lambda_{\text {em }}=630 \mathrm{~nm}\right.$ band pass $)$. The existence of a minor additional protonated species with a lifetime of $\sim 4.0 \mathrm{~ns}$ ( $\lambda_{\mathrm{ex}}=526 \mathrm{~nm}, \lambda_{\mathrm{em}}=545 \mathrm{~nm}$ long pass $)$ is also conceivable.

Photometric considerations conclude the examination of PAc-SNARF in this section. The $\mathrm{S}_{1} \leftarrow \mathrm{S}_{0}$ absorption band is available in terms of lognormal functions of wavenumber and
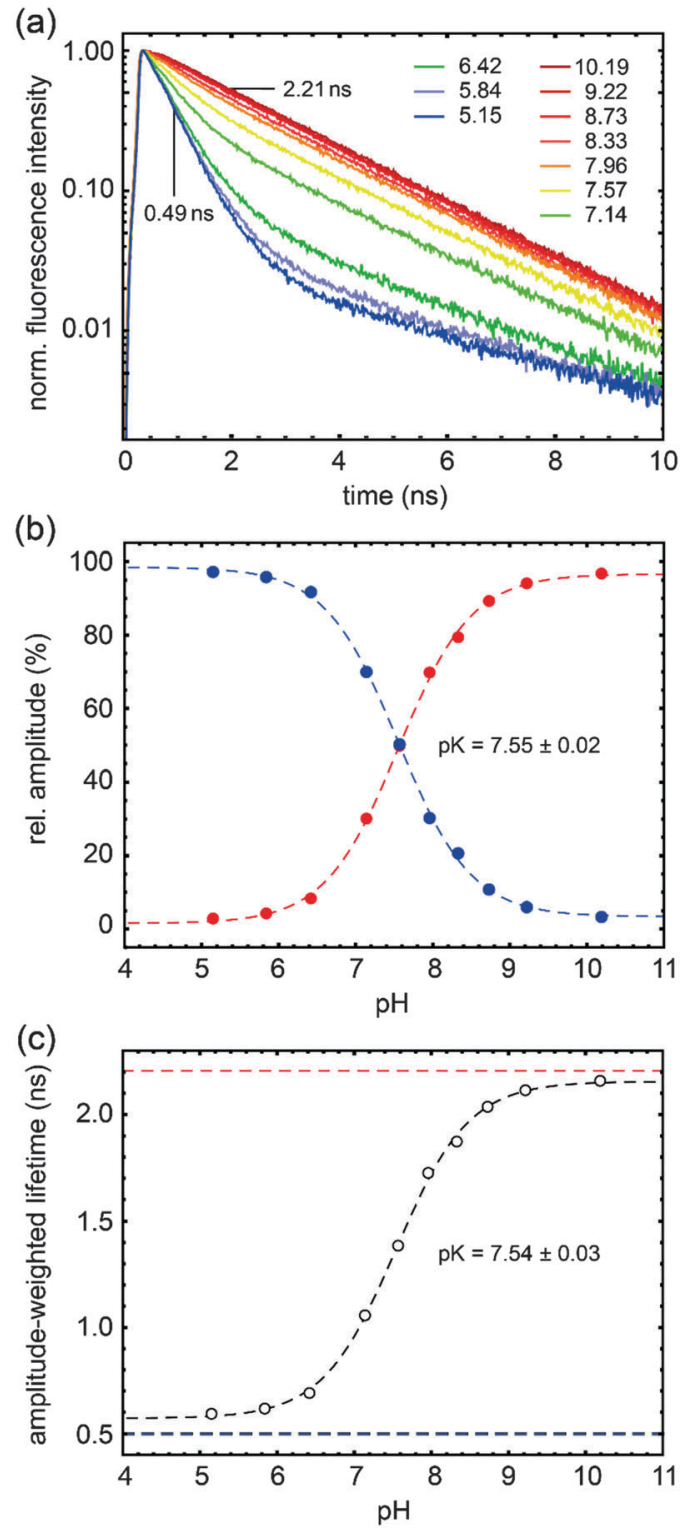

Fig. 2 Time-resolved fluorescence of PAc-SNARF as a function of $\mathrm{pH}$. (a) Decay traces at various $\mathrm{pH}$ values as indicated by the different colors. Red and blue curves refer to the pure deprotonated and protonated species; the two lifetimes from global analysis are indicated. (b) Relative amplitudes of the decay components due to the protonated (blue) and deprotonated form (red curve). (c) Amplitude-weighted lifetimes. Fit lines and $\mathrm{p} K_{\mathrm{a}}$ values are also given. Conditions: $10 \mathrm{mM}$ Tris Citrate, $190 \mathrm{mM} \mathrm{NaCl}, 20: 1$ water:dmso volume fraction, $20^{\circ} \mathrm{C}$. $\lambda_{\text {exc }}=526 \mathrm{~nm}$, and emission was detected with long pass filter $\mathrm{HQ} 545$.

can therefore be integrated entirely (12 000-25000 $\left.\mathrm{cm}^{-1}\right)$. Thus we find oscillator strengths $f_{\text {osc,blue }}=0.65$ and $f_{\text {osc,red }}=0.83$. Radiative lifetimes are calculated with the Strickler-Berg formula, resulting in $\tau_{\text {rad,blue }}=6.03 \mathrm{~ns}$ and $\tau_{\text {rad,red }}=5.08 \mathrm{~ns}$. Compare this with the measured fluorescence lifetimes of $\tau_{\text {blue }}=0.49 \mathrm{~ns}$ and $\tau_{\text {red }}=2.21$ ns (Fig. 3). Looking only at the red form at $\mathrm{pH}>9$, we predict a fluorescence quantum yield $\mathrm{QY}_{\text {red }}=44 \%$. Instead our direct measurement (with rhodamine 101/methanol as reference) gave a value of $35 \%$. The experimental value being lower is 
(a)

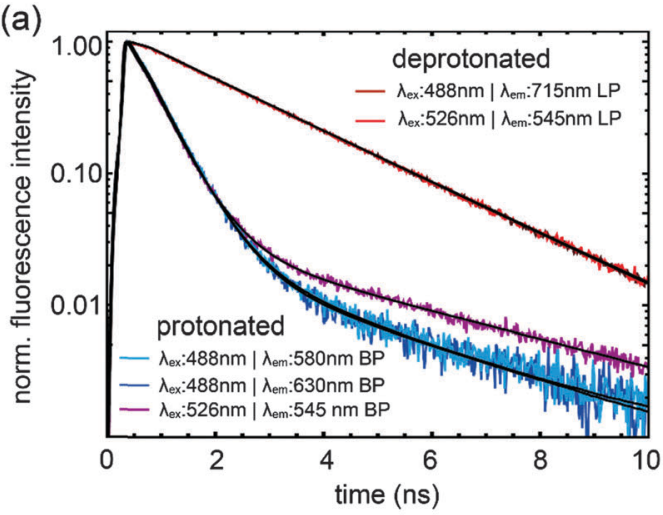

(b)

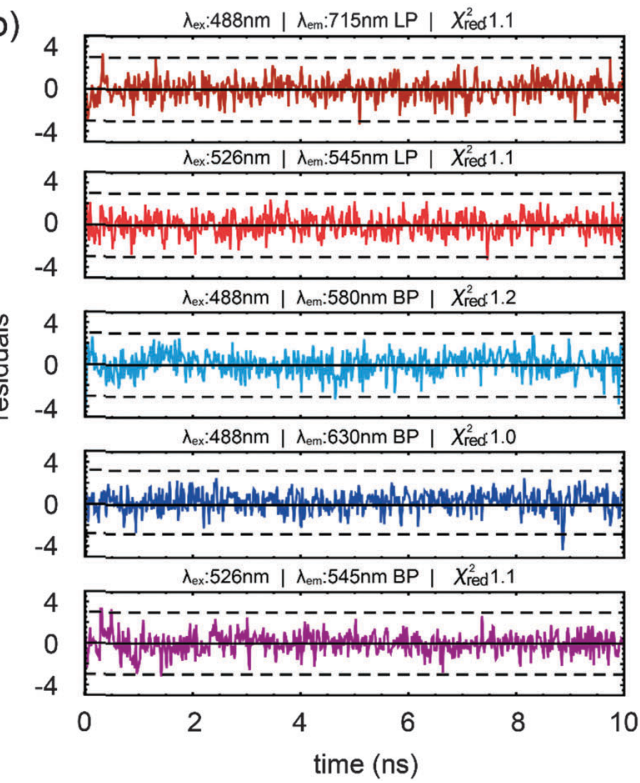

Fig. 3 Fluorescence decay analysis of protonated and deprotonated forms of PAC-SNARF for different excitation and emission wavelengths, as indicated. (a) Fluorescence decay traces and their multi-exponential fits. (b) Fit residuals. (BP-band pass; LP-long pass filter).

consistent with the idea that a higher electronically excited state contributes to the first absorption band of the red form.

Quantum-chemical results for the SNARF core (i.e. by setting $\mathrm{R}=\mathrm{H}$, see Scheme 2) in water are presented in Table 2. The lowest-energy transition of the protonated form is calculated at $\Delta E=21290 \mathrm{~cm}^{-1}$ with oscillator strength $f=0.49$. It is accompanied by a moderate change in dipole moment, $\Delta \mu=4.3 \mathrm{D}$ (=Debye). For the deprotonated form the lowest transition at $20080 \mathrm{~cm}^{-1}$ is predicted to be strong $(f=0.75)$. The energy separation of the lowest excited electronic states and the relative strengths of the corresponding transitions are in good agreement with observations. Note that the TDDFT method also finds a charge transfer transition ( $\Delta \mu=25.1$ Debye) with zero oscillator strength. This is due to a well-known artifact of DFT methods which overestimate the stability of charge-transfer states. Molecular orbitals are shown in the ESI. $\dagger$

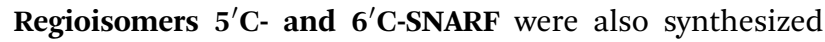
and isolated. The dependence of optical spectra on $\mathrm{pH}$ was examined in water:dmso $5: 1$ in this case, in order to avoid
Table 2 Vertical excitation energy $\Delta E$, oscillator strength $f$, and dipole moment change $\Delta \mu=\left|\mu_{i}-\mu_{0}\right|$ of the SNARF core $(\mathrm{R}=\mathrm{H})$ in water, calculated with TD-DFT/B3LYP/cc-pVDZ for the first three electronic transitions

\begin{tabular}{lllr}
\hline & $\Delta E / \mathrm{cm}^{-1}$ & $f$ & $\Delta \mu / \mathrm{D}$ \\
\hline Deprotonated & 20080 & 0.752 & 7.43 \\
& 22460 & 0.000 & 25.12 \\
& 23910 & 0.008 & 6.96 \\
Protonated & 21290 & & \\
& 25140 & 0.491 & 4.27 \\
& 27130 & 0.158 & 10.99 \\
& & 0.027 & 8.01 \\
\hline
\end{tabular}

solubility problems at $\mathrm{pH} \sim 5$. Species-associated spectra of absorption and fluorescence are almost identical to those of PAc-SNARF at the same cosolvent concentration; however the $\mathrm{p} K_{\mathrm{a}}$ of $5^{\prime} \mathrm{C}$ - is larger by 0.15 compared to $6^{\prime} \mathrm{C}$ - (see $\mathrm{ESI} \dagger$ ). In precision measurements of $\mathrm{pH}$ it is important to use only one of the isomers, because if a mixture is used, protonated and deprotonated forms coexist in a broader range and resolution is lost correspondingly.

\subsection{Labelling of cyanobacterial phytochrome Cph1 $\Delta 2$}

The function of light-activated proteins such as bacteriorhodopsin, visual rhodopsin, channelrhodopsin or phytochrome is associated with protonation changes in the protein and subsequent proton release/uptake events. ${ }^{32} \mathrm{~A}$ pH-dependent dye for covalent linkage to the protein surface often used in the investigation of these proteins is iodoacetamido-fluorescein, IA-F. ${ }^{33}$ Instead we test here the covalent binding of IA-SNARF to a protein surface, using the cyanobacterial phytochrome $\operatorname{Cph} 1 \Delta 2^{30}$ as example.

Under the conditions described in the Experimental Section, about $0.8 \mathrm{~mol}$ of IA-SNARF was bound per mol Cph1 $\Delta 2$. The 2VEA crystal structure ${ }^{29}$ implies that only C371 in the sensor module is exposed and thus is the most likely target for the label. Fig. 4 shows the absorption spectra of Cph1 $\Delta 2$ in the Pr form of phytochrome. For $\mathrm{pH} 6$ and $\mathrm{pH} 8.8$, spectra before and after labelling are compared (dashed and solid line, respectively). When SNARF is bound to the protein, the absorption band has its maximum at $\lambda_{\max }=529 \pm 1 \mathrm{~nm}$ in the protonated form and at $\lambda_{\max }=565 \pm 1 \mathrm{~nm}$ in the deprotonated form. The protonated form is slightly red-shifted compared to that of PAc-SNARF (cf. Fig. 1a). The same holds true for the isosbestic point, which is now observed at $541 \mathrm{~nm}$. The fluorescence lifetimes of IA-SNARF are $\tau_{\text {blue }}=0.49 \mathrm{~ns}$ and $\tau_{\text {red }}=2.10 \mathrm{~ns}$, very similar to PAc-SNARF. As the peak absorbance of phytochrome in the Pr form is located at $658 \mathrm{~nm}$, an excitation of the SNARF dye results in minimal phytochrome activation. This is one prerequisite for using the SNARF dye with photoreceptor proteins.

\section{Outlook}

Besides investigations on the molecular level, monitoring the $\mathrm{pH}$ inside living cells is important since intracellular $\mathrm{pH}$ changes are critical for cell and tissue activities, including phagocytosis, 
(a)

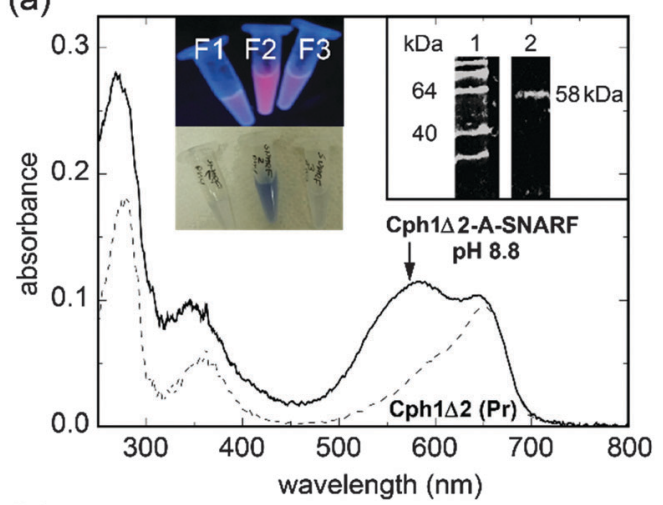

(b)

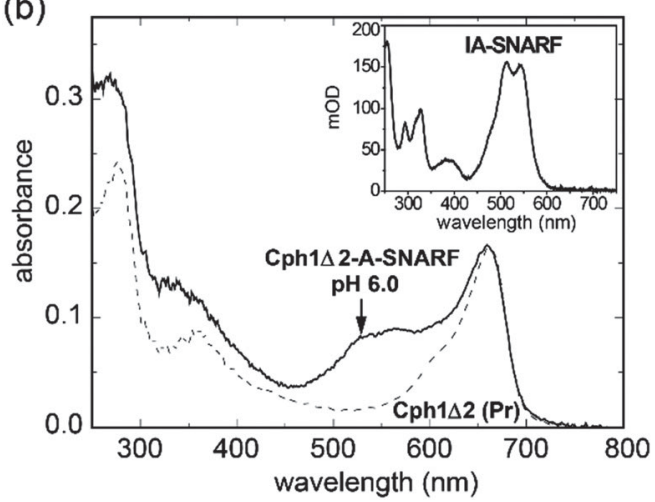

Fig. 4 Covalent binding of IA-SNARF to cyanobacterial phytochrome Cph1 $\triangle 2$. Absorption spectra are shown before (- - -) and after (-) labelling at $\mathrm{pH} 8.8$ (a) and $\mathrm{pH} 6.0$ (b). Arrows indicate the absorption maximum of the deprotonated $(565 \mathrm{~nm}$ at $\mathrm{pH} 8.8$ ) and the protonated $(529.5 \mathrm{~nm}$ at $\mathrm{pH} 6)$ form of bound SNARF. Inset in (a): left - photographs of the fractions after gel filtration containing SNARF (red fluorescence in fraction F2) and phytochrome (blue absorption in fraction F2); right - SDS PAGE of IA-SNARF in the fluorescence image (lane 1: molecular weight marker, lane 2: Cph1 $\Delta 2-$ A-SNARF). Inset in (b): absorption spectrum of IA-SNARF at pH 6.0.

endocytosis, ion transport, signaling cascades, and synaptic transmission. Both PAc-SNARF and IA-SNARF are able to enter the cell freely.

To validate the applicability of our SNARF derivatives as dual fluorescence probe for quantitative $\mathrm{pH}$ measurements in imaging experiments, we conducted two proof-of-principle experiments using a confocal raster scanning fluorescence microscope equipped with TCSPC detection for fluorescence lifetime experiments. ${ }^{29}$

The first experiment (see Fig. 5) demonstrates the amplitudeweighted-lifetime method for determining $\mathrm{pH}$. Two drops of PAcSNARF solution with different $\mathrm{pH}$ values (6.5 and 7.8) are placed on a cover slip. Panel (a) shows the false-coded FLIM image according to the different fluorescence lifetime characteristics of the image, clearly indicating the two different drops in the image. The corresponding fluorescence decay curves and lifetimes are shown in panel (b). The calibration curve from the TCSPC measurements (Fig. 2c) was used to obtain the pH values as shown in Fig. 5c, which match the target $\mathrm{pH}$ values. Since the lifetime is used in the pH calibration curve, it does not matter whether the calibration was obtained in a spectroscopic or microscopic setup as long as the excitation and emission parameter are identical. (a)

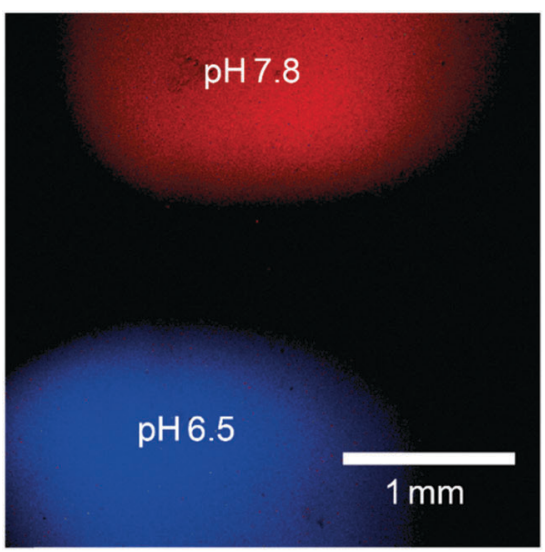

(b)

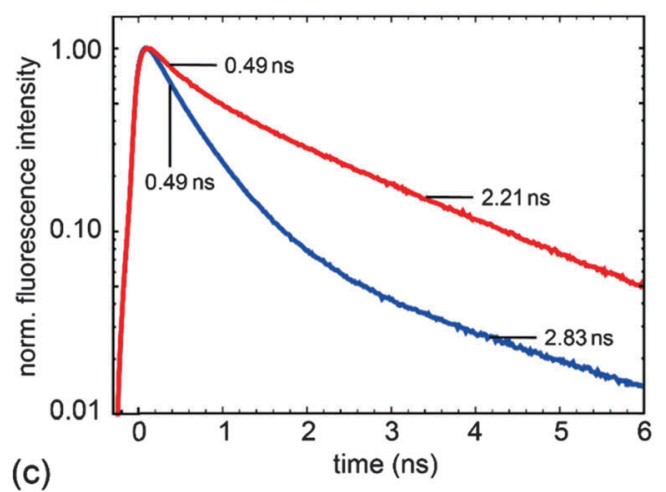

(c)

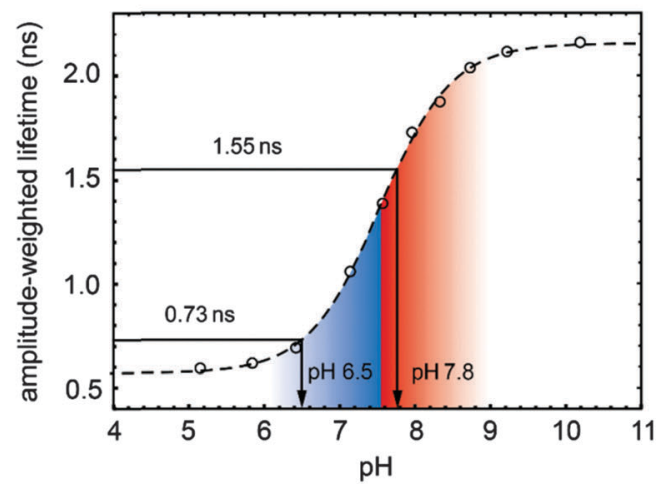

Fig. 5 FLIM based determination of $\mathrm{pH}$ values. (a) FLIM image of PAcSNARF in acidic (blue) and basic (red) environment. Conditions: $4 \times$ objective, excitation at $526 \mathrm{~nm}$ and emission filter HQ545LP. (b) Fluorescence decay traces of protonated (blue) and deprotonated (red) PAc-SNARF of (a). (c) pH calibration curve obtained by TCSPC cuvette measurements using the same excitation wavelength and emission filter. The determined amplitudeweighted lifetimes ( $0.73 \& 1.55 \mathrm{~ns})$ of the traces in (b) and respective determined $\mathrm{pH}$ values (6.5 \& 7.8) are shown.

The second experiment (see Fig. 6) demonstrates the ratiometric-yield method with a SNARF-labelled protein. Here the two emission bands from only one excitation wavelength are exploited. Using the standard set of emission filters that exists in many confocal laser scanning microscopes, we measure the stationary fluorescence intensity of the SNARF-labelled phytochrome in the "red" and "green" emission channel (green: $\lambda_{\mathrm{em}}=$ $545-560 \mathrm{~nm}$, red: $\lambda_{\mathrm{em}}>590 \mathrm{~nm}$ ). The corresponding images are shown in panel (a). The calibration curve (b) was obtained with 
(a)

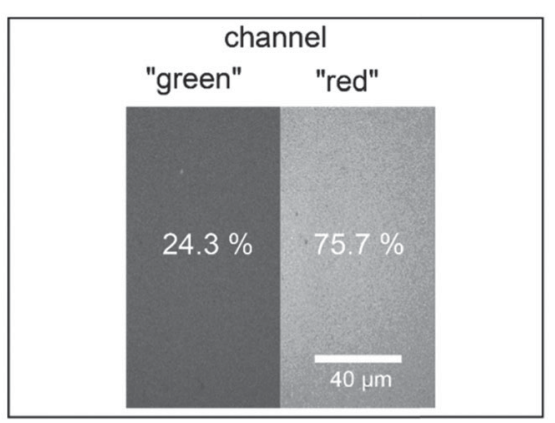

(b)

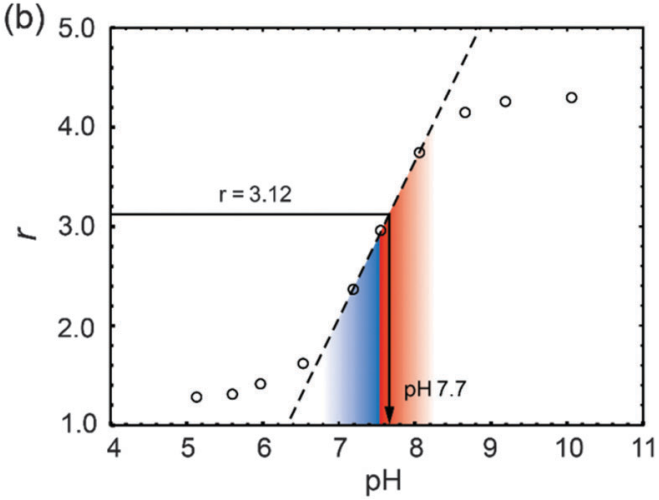

Fig. 6 Laser scanning image for ratiometric intensity based determination of $\mathrm{pH}$ values. (a) Image of SNARF-labelled phytochrome in the two detection channels. Conditions: $40 \times$ objective, excitation at $526 \mathrm{~nm}$ and dual detection mode using a dichroic filter $560 \mathrm{dclp}$. In the "green" and "red" channel an additional HQ545LP respectively OG590 longpass filter was used. (b) $\mathrm{pH}$ calibration curve obtained by the fluorescence intensity ratio $r=I_{\text {red }} / I_{\text {green }}$ of both channels measuring PAC-SNARF calibration samples at different $\mathrm{pH}$ values.

the confocal microscope using the same settings as for the ratiometric $\mathrm{pH}$ determination of the $\mathrm{pH}$ at the protein surface of phytochrome. The resulting $\mathrm{pH}$ value is 7.7.

The results of these proof-of-principle experiments show clearly that the new SNARF dye offers the opportunity for tracking spatio-temporal $\mathrm{pH}$ changes inside living cells, and to elucidate the function of proteins to which the SNARF dye is attached to.

\section{Acknowledgements}

This project was supported by the Deutsche Forschungsgemeinschaft through grants to UA and NPE (Collaborative Research Center 1078 "Protonation Dynamics in Protein Function", projects A2 and A3) and to UA (Collaborative Research Center 1112 "Nanocarriers: Architecture, Transport, and Topical Application of Drugs for Therapeutic Use”, project B03). We would like to thank Dr Katharina Achazi for providing HeLa cells.

\section{Notes and references}

1 J. Han and K. Burgess, Chem. Rev., 2010, 110, 2709-2728.

2 A. M. Paradiso, R. Y. Tsien and T. E. Machen, Proc. Natl. Acad. Sci. U. S. A., 1984, 81, 7436-7440.
3 X. Wang, A. J. Levi and A. P. Halestrap, Am. J. Physiol.: Heart Circ. Physiol., 1994, 267, H1759-H1769.

4 M. J. Fehr, M. A. McCloskey and J. W. Petrich, J. Am. Chem. Soc., 1995, 117, 1833-1836.

5 R. P. Haugland and J. Whitaker and U.S. Pat., 4945171, 1990.

6 J. Liu, Z. Diwu and W.-Y. Leung, Bioorg. Med. Chem. Lett., 2001, 11, 2903-2905.

7 E. Nakata, H. Wang and I. Hamachi, ChemBioChem, 2008, 9, 25-29.

8 A. Keppler, H. Pick, C. Arrivoli, H. Vogel and K. Johnsson, Proc. Natl. Acad. Sci. U. S. A., 2004, 101, 9955-9959.

9 E. Nakata, Y. Nazumi, Y. Yukimachi, Y. Uto, H. Maezawa, T. Hashimoto, Y. Okamoto and H. Hori, Bioorg. Med. Chem. Lett., 2011, 21, 1663-1666.

10 R. Martinez-Zaguilan, G. M. Martinez, F. Lattanzio and J. R. Gillies, Am. J. Phys., 1991, 260, 297-307.

11 M. Frasconi, R. Tel-Vered, J. Elbaz and I. Willner, J. Am. Chem. Soc., 2010, 132, 2029-2036.

12 M. A. Schroeder, M. A. Ali, A. Hulikovam, C. T. Supuran, K. Clarke, R. D. Vaughan-Jones, D. J. Tyler and P. Swietach, Proc. Natl. Acad. Sci. U. S. A., 2013, 110, E958-E967.

13 M. P. Weekes, S. Y. Tan, E. Poole, S. Talbot, R. Antrobus, D. L. Smith, C. Montag, S. P. Gygi, J. H. Sinclair and P. J. Lehner, Science, 2013, 340, 199-202.

14 S. Schlafer, J. E. Garcia, M. Greve, M. K. Raarup, B. Nyvad and I. Dige, Appl. Environ. Microbiol., 2015, 81, 1267-1273.

15 K. J. Buckler and R. D. Vaughan-Jones, Pflugers Arch., 1990, 417, 234-239.

16 M. V. Kvach, D. A. Tsybulsky, A. V. Ustinov, I. A. Stepanova, S. L. Bondarev, S. V. Gontarev, V. A. Korshun and V. V. Shmanai, Bioconjugate Chem., 2007, 18, 1691-1696.

17 I. Johnson and M. T. Z. Spence, The Molecular Probes Handbook, 11th edn, 2010, ch 1.5, Fluorescein, Oregon Green and Rhodamine Green Dyes.

18 Y. Kaneko and D. C. Neckers, J. Phys. Chem. A, 1998, 102, 5356-5363.

19 J. Karpiuk, J. Phys. Chem. A, 2004, 108, 11183-11195.

20 J. Karpiuk, Z. R. Grabowski and F. C. De Schryver, J. Phys. Chem., 1994, 98, 3247-3256.

21 A. Galant, R. P. Koester, E. A. Ainsworth, L. M. Hicks and J. M. Jez, New Phytol., 2012, 194, 220-229.

22 T. Kreisig, R. Hoffmann and T. Zuchner, Anal. Chem., 2011, 83, 4281-4287.

23 M. Misheva, G. Kaur, K. R. W. Ngoei, Y. Y. Yeap, I. H. W. Ng, K. M. Wagstaff, D. C. H. Ng, D. A. Jans and M. A. Bogoyevitch, Biochim. Biophys. Acta, 2014, 1843, 253-264.

24 A. A. Granovsky, GAMESS Firefly version 8, (http://classic. chem.msu.su/gran/firefly/index.html) which is partly based on, M. W. Schmidt, K. K. Baldridge, J. A. Boatz, S. T. Elbert, M. S. Gordon, J. H. Jensen, S. Koseki, N. Matsunaga, K. A. Nguyen, S. Su, T. L. Windus, M. Dupuis, J. A. Montgomery, J. Comput. Chem., 14, 1993, 1347-1363.

25 K. H. Drexhage, J. Res. NBS, Sect. A: Phys. Chem., 1976, 80, 421-428.

26 U. Alexiev, I. Rimke and T. Pöhlmann, J. Mol. Biol., 2003, 328, 705-719. 
27 T.-Y. Kim, K. Winkler and U. Alexiev, Photochem. Photobiol., 2007, 83, 378-385.

28 U. Alexiev and D. L. Farrens, Biochim. Biophys. Acta, Bioenerg., 2014, 1837, 694-709.

29 A. Boreham, T.-Y. Kim, V. Spahn, C. Stein, L. Mundhenk, A. D. Gruber, R. Haag, P. Welker, K. Licha and U. Alexiev, ACS Med. Chem. Lett., 2011, 2, 724-728.

30 L. O. Essen, J. Mailliet and J. Hughes, Proc. Natl. Acad. Sci. U. S. A., 2008, 105, 14709-14714.
31 The storage form (see ESI $\dagger$ ) was weighed in since it can be purified better. Reproducible results were obtained when milligram quantities were dissolved directly in the appropriate final liquid volume.

32 J. J. van Thor, B. Borucki, W. Crielaard, H. Otto, T. Lamparter, J. Hughes, K. J. Hellingwerf and M. P. Heyn, Biochemistry, 2001, 40, 11460-11471.

33 U. Alexiev, P. Scherrer, T. Marti, H. G. Khorana and M. P. Heyn, FEBS Lett., 1995, 373, 81-84. 